\title{
Retinal tear extension through the cryosurgical scar
}

WILLIAM V. DELANEY, JR.

From the Department of Ophthalmology, State University of New York Upstate Medical Center

The popularity of retinal cryopexy is increasing (Lincoff, McLean, and Nano, 1964). Some workers (Havener and Gloeckner, 1967) indicate that anything less is obsolete. The advantages claimed for it are intact sclera, lower infection rate, and less vitreous damage. Disadvantages include clumsy instruments, risk of choroidal haemorrhage and the difficulty of working with invisible applications.

Shea (1968), however, mentioned the possibility of extension of retinal breaks after cryosurgery. Six of I I 7 consecutive patients treated prophylactically by the author with cryosurgery had vitreous haemorrhage due to extension of retinal tears. This compares unfavourably with the absence of haemorrhage following photocoagulation for similar disease in 74 patients. The discrepancy warrants attention.

\section{Gryopexy technique}

All patients were treated trans-sclerally or transconjunctivally by the Linde or Amoils cryoprobe under observation by the indirect ophthalmoscope (Fig. I). The margins of each freeze overlapped around the outline of the tear. Additional applications were made at the base of the operculum. A second ring of non-overlapping lesions was placed outside the first and always brought to the ora serrata. Applications on the tear edge were continued until slight whitening of the retina occurred. The second row lesions were limited to freezing the choroid. Treatment of lattice degeneration consisted of coalescent freezing in full-thickness retina around weak areas with a second row of applications peripheral to it. One patient had a scleral imbrication to create a small buckle. None of the patients

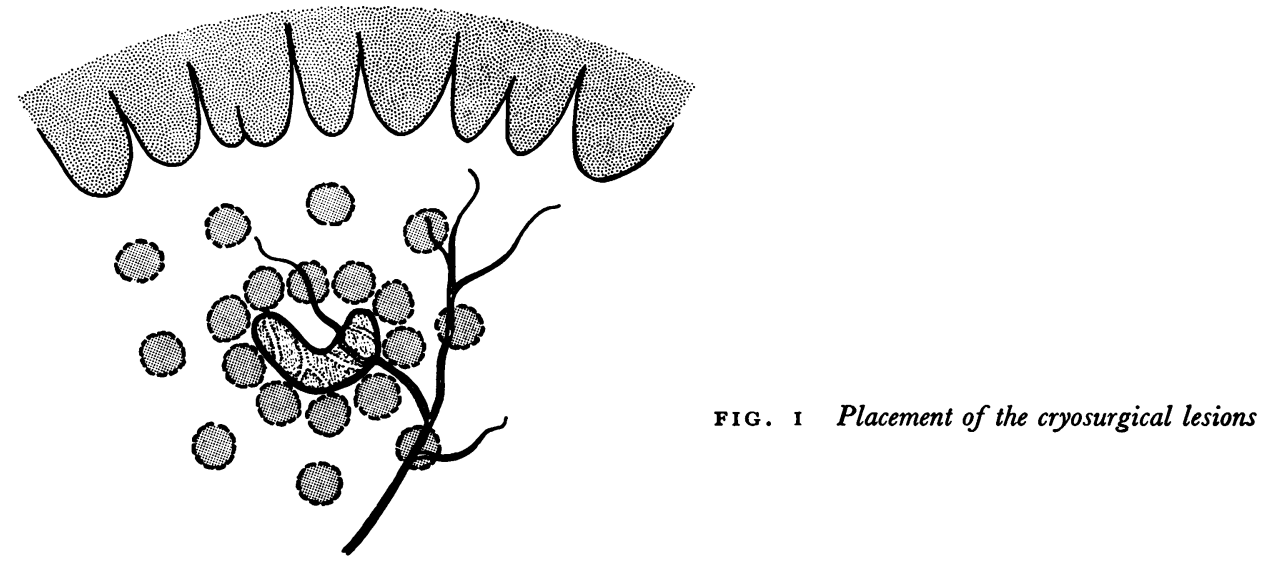


had frank retinal detachment. All had vitreous haemorrhage and all had tears superior to the long posterior ciliary nerves. All patients achieved a well-pigmented scar around్ㅡㅁ the retinal tear in the expected time and before the subsequent bleeding (Table).

Table Summary of results in six cases

\begin{tabular}{|c|c|c|c|c|c|c|}
\hline Case No. & Sex & $\begin{array}{l}\text { Age } \\
\text { (yrs) }\end{array}$ & Operation & $\begin{array}{l}\text { Time of re-bleeding } \\
\text { after initial treatment } \\
\text { (mths) }\end{array}$ & Additional treatment & $\begin{array}{l}\text { Final } \\
\text { visual } \\
\text { acuity }\end{array}$ \\
\hline 2 & $\mathbf{M}$ & 65 & Trans-conj. cryopexy & 2 & None & $20 / 20$ \\
\hline 3 & $\mathbf{F}$ & $5^{1}$ & Trans-conj. cryopexy & $\begin{array}{r}8 \\
12 \\
14\end{array}$ & $\begin{array}{l}\text { Further cryopexy } \\
\text { None } \\
\text { Diathermy and local } \\
\text { buckle }\end{array}$ & $20 / 30$ \\
\hline 4 & $\mathbf{F}$ & 57 & Trans-conj. cryopexy & $\begin{array}{r}4 \\
7 \\
10\end{array}$ & $\begin{array}{l}\text { Further cryopexy } \\
\text { None } \\
\text { None }\end{array}$ & $20 / 40$ \\
\hline 5 & $\mathbf{F}$ & 65 & Trans-conj. cryopexy & 4 & $\begin{array}{l}\text { Local buckle and } \\
\text { cryopexy }\end{array}$ & $20 / 25$ \\
\hline 6 & $\mathbf{M}$ & $5^{6}$ & Trans-conj. cryopexy & $\begin{array}{l}6 \text { (Cataract surgery } \\
\text { in interim) }\end{array}$ & None & $20 / 20$ \\
\hline $\mathbf{I}$ & $\mathbf{M}$ & 64 & $\begin{array}{l}\text { Cryopexy and } \\
\text { scleral imbrication }\end{array}$ & 7 & Laser photocoagulation & $20 / 20$ \\
\hline
\end{tabular}

\section{Summary of case histories}

(I) A 64-year-old white male had a surge of "green seaweed" in the right eye 3 months after bilateral cataract surgery. His vision was reduced to $20 / 50$ and a retinal tear was observed at

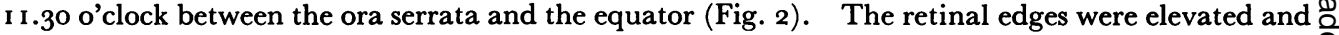
the operculum was vertical in the vitreous. Cryopexy with a scleral imbrication for a local buckle $\mathbb{Q}$ was done. The buckle gradually disappeared, but 7 months later the patient complained of a $\overrightarrow{0}$ sudden "cloud" in the same eye. Vitreous blood was again present and an extension of the original 3 tear anteriorly through a well-pigmented cryosurgical scar was shown by small retinal haemorrhages at the margins. No other holes or tears could be found. Laser photocoagulation was used to supplement treatment.

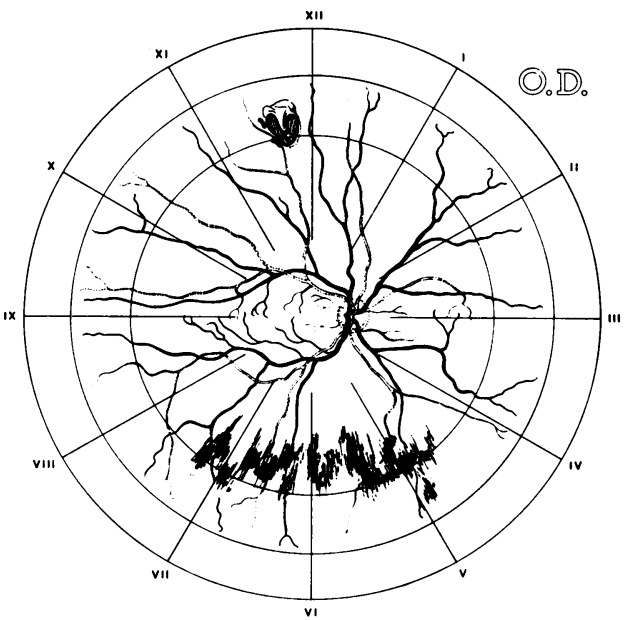

FIG. 2 Patient I

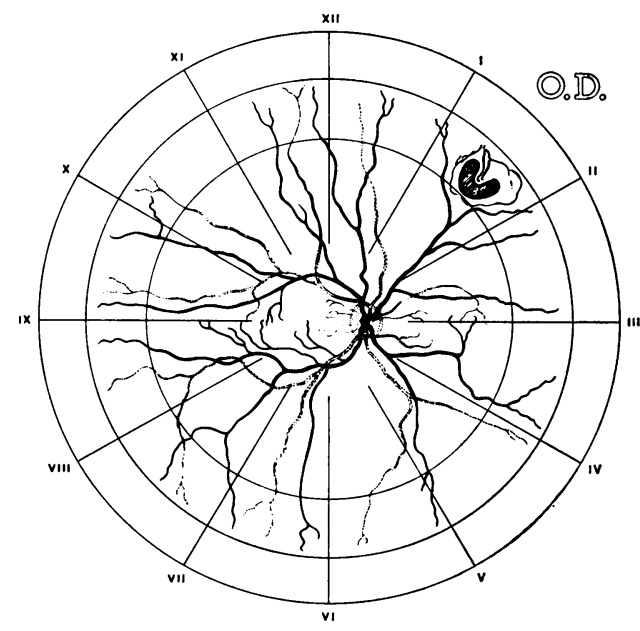

FIG. 3 Patient 2 
(2) A 65-year-old white male myope had extraction of a hypermature cataract and recovered $20 / 20$ vision in the right eye. After 20 months a retinal tear was discovered at 2.30 o'clock near the ora serrata on routine examination (Fig. 3). Transconjunctival cryopexy was done and a good scar was achieved in one week, but 2 months later vitreous haemorrhage reduced vision to 20/60. No other retinal breaks could be found. The original tear appeared to have extended anteriorly but only within the cryosurgically scarred area. No treatment was given. The visual acuity I year later was $20 / 20$.

(3) A 5 I-year-old white female had a sudden vitreous haemorrhage in the left eye which reduced vision to 20/400. Multi-level lattice degeneration was present across both superior quadrants of both eyes (Fig. 4). Tears at both ends of the lattice in each eye prompted bilateral transconjunctival cryopexy. The vision returned to 20/30 in the left eye and the right maintained 20/25. After 8 months the patient noted "smoke" in the left eye. The large superior nasal tear had extended anteriorly as indicated by marginal retinal haemorrhages. New retinal breaks were absent. Further cryopexy was placed; 4 months later bleeding recurred from the same area, and 2 months later the episode repeated. 19 months after the original bleeding, diathermy and local buckling were done. One year later the visual acuity was $20 / 30$ and no further bleeding had occurred.

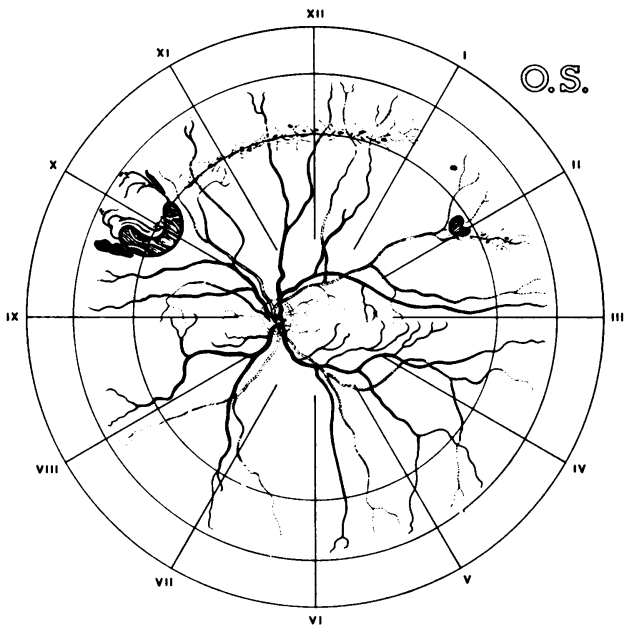

FIG. 4 Patient 3

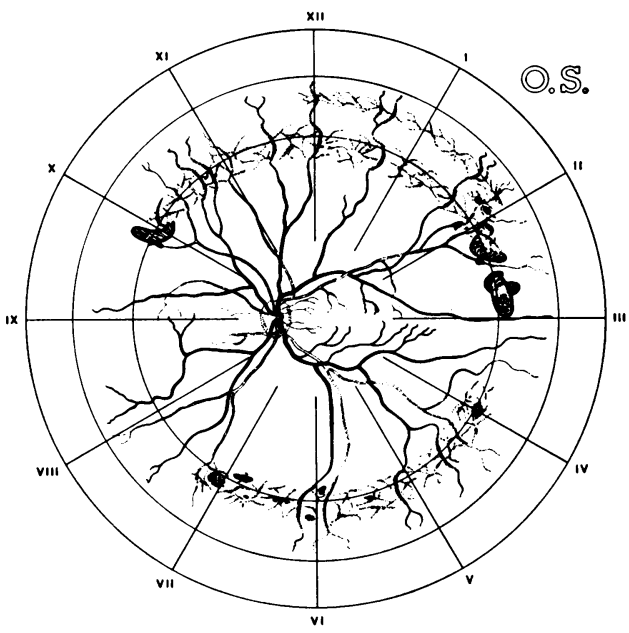

FIG. 5 Patient 4

(4) A 57-year-old white female myope had had "smoke" in the left eye for 2 months before examination. Vision was 20/20 in both eyes. Multilevel lattice degeneration bridged both superior and inferior quadrants of both eyes (Fig. 5). The left eye had tears at both ends of the superior degeneration. Transconjunctival cryopexy was done, but 4 months later vitreous haemorrhage recurred in the left eye despite coalescent scarring around the retinal breaks and all lattice. More cryopexy was placed, but 3 months later another haemorrhage was associated with obvious extension of the supero-nasal tear. The patient was found to have hypertension and was treated. No new tears occurred and bleeding stopped. No further treatment was given. The retina remained attached 3 months later although the vitreous was hazy.

(5) A 65-year-old white female had noted "floaters" for one month before examination. Vision in the right eye was 20/20, and in the left hand movements. Open-angle glaucoma was present in the right eye and the left had a total retinal detachment. The history indicated that the left eye was amblyopic. Surgery improved vision to 20/200. Pilocarpine I per cent. controlled the glaucoma in the right eye but $\mathrm{I}$ month after starting pilocarpine the patient had flashes and a tear was found at II o'clock at the equator (Fig. 6). Transconjunctival cryopexy gave good scars; 4 months later a new tear occurred beside the original but within the treatment scars. Slight retinal 
elevation prompted the application of further transscleral cryopexy and a trap-door buckle. The vision was 20/25 3 months later.

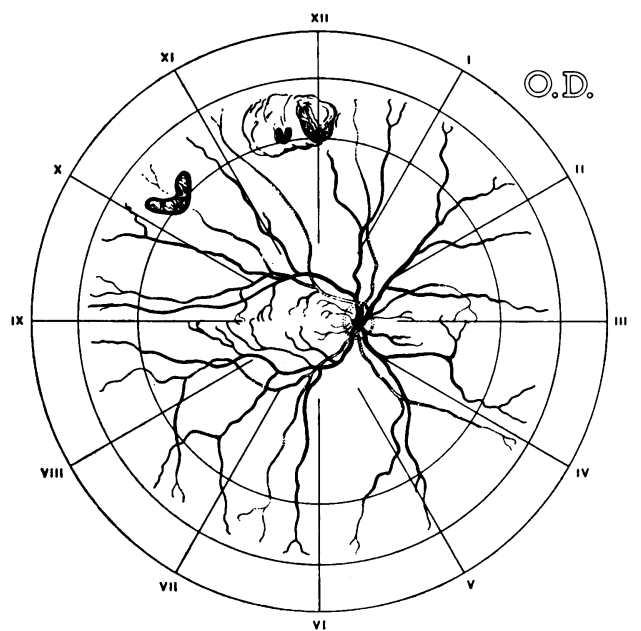

FIG. 6 Patient 5

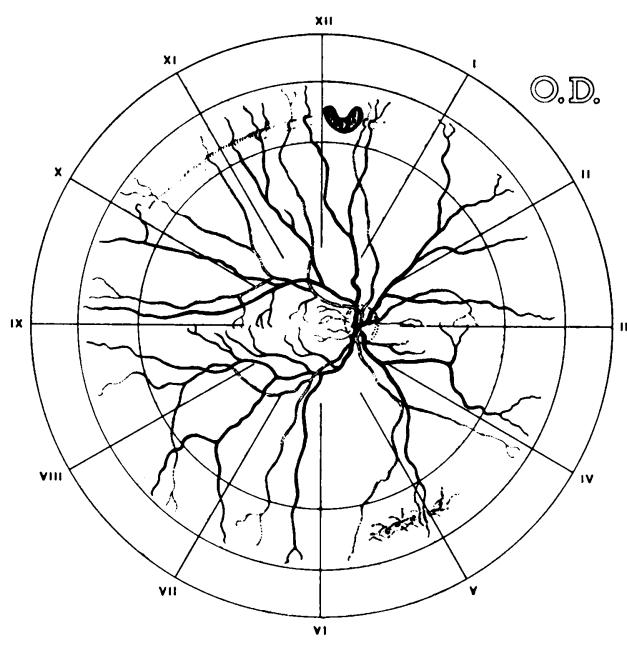

FIG. 7 Patient 6

(6) A 56-year-old white male, a mild myope, had had a cataract extraction on the left eye $4 \frac{1}{2}$ months before a retinal detachment. Retinal surgery resulted in 20/20 vision. A cataract reduced vision in the right eye to 20/100 3 years later. Before cataract surgery on this eye the patient developed flashes and a small tear was found at 12 o'clock (Fig. 7). Transconjunctival cryopexyo was done, followed in 6 months by cataract extraction; 2 weeks after surgery the patient had flashes and only a red reflex could be obtained. The vitreous cleared and the eye was qniet. Expectedo tears could not be found but small haemorrhages at the margins indicated an extension of the new original tear within the cryosurgical scar. No treatment was given. After 3 months flashes recurred and a new hole was found at 3 o'clock. Laser photocoagulation was used. The vision was 20/20 6 months later.

\section{Results}

In six of a series of I I 7 patients retinal tears extended within visible and well-establishedo․ cryosurgical scars. All six bled into the vitreous, but none had a retinal detachment, and $\frac{5}{3}$ each retained useful vision. Two had local buckling surgery, one responded to further cryopexy alone, and one to photocoagulation, and two had no additional treatment (Table). Of a further series of 74 patients treated prophylactically with photocoagulation, $\frac{9}{2}$ none had a similar episode.

\section{Discussion}

Anatomical facts confirm that vitreous attachment is stronger along retinal blood vessels. Clinical verification comes from the frequent association of vitreous haemorrhage with retinal tears. Almost every tear crosses a blood vessel and four of the six patients presented above demonstrate this fact. In practice, if an operculated tear extends within photocoagulation or diathermy scars, vitreous haemorrhage and retinal detachment are uncommon.

Those who support the use of cryopexy (Lincoff, and others 1964; Lincoff and McLean, 0 1966; McPherson, 1968) agree that choroidal and retinal blood vessels are spared throm- $\frac{O}{\Phi}$ bosis by this procedure. Likewise, Bruch's membrane is left intact (Lincoff and McLean, $\stackrel{?}{\mathbb{P}}$ 1969; Amoils and Honey, 1969). Unlike diathermy and photocoagulation (Kissen, 
Delaney, and Wachtel, I96I), this vascular sparing allows contiguous placement of cryosurgical lesions. Despite this metabolic chorio-retinal advantage, intact blood vessels may lead to functional failure from haemorrhage if unrelieved vitreous traction extends the tear.

Accurate knowledge of the tensile strength of heat or cold chorioretinal scars escapes us. Vague ideas exist that increased cold increases strength (Lincoff, Long, Marquardt, and McLean, i 968). If adjacent position, longer duration, and lower temperature can increase lesion strength, the patients herein described show the need for more attention to the hinged edge of a retinal operculum. This adds another reason, apart from accuracy, for visualizing each cryosurgical lesion (Lincoff and McLean, ig69).

Post-operative pain, phthisis, anterior segment necrosis (Duguid, 1967), and implant erosion may follow raised intraocular pressure due to scleral buckling. Large areas of lattice degeneration with multiple retinal breaks often cannot be effectively relieved of traction without risk in the absence of subretinal fluid, even with paracentesis or osmolar therapy to lower the intraocular pressure.

Possible tear extension through the cryosurgical scar makes an indentation reassuring. However, indentations without an encircling element are nearly always externalized and thereby lose their effectiveness in preventing tear extension. The permanency of an encircling element, despite the risk of raised intraocular pressure, must be considered carefully in light of the patients described here if cryotherapy is used.

Transconjunctival cryotherapy for threatening retinal breaks is an advantage not to be ignored. The presence of cataract, vitreous blood, and corneal scars, which prohibit photocoagulation, make it invaluable. While it simplifies the therapy of anterior retinal breaks, it is not a panacea. Those who undertake retinal cryotherapy should be aware of its weaknesses and be prepared to place photocoagulation, a permanent indentation, or diathermy if tear extension through the cryosurgical scar threatens vision with detachment or vitreous blood.

\section{Summary}

Six patients are presented who had vitreous haemorrhage due to the extension of retinal tears through well-established cryosurgical scars. All maintained useful vision. Two had supplemental trap-door scleral buckling, one needed further cryopexy, and one responded to photocoagulation. Two had no additional therapy. Awareness of this possible inherent weakness of cryosurgical scars is essential.

\section{References}

AMOILs, S. P., and HONEY, D. P. (1969) Arch. Ophthal. (Chicago), 82, 220

DUGUID, I. м. (1967) Trans. ophthal. Soc. U.K., 87, I 7 I

haVENER, W. H., and Gloeckner, s. (1967) "Atlas of Diagnostic Techniques and Treatment of

Retinal Detachment", p. ı08, Mosby, St. Louis

kissen, A. T., Delaney, w. v., JR., and Wachtel, J. (196i) Amer. F. Ophthal., 52, 487

lincoff, h. A., long, R., marguardt, J., and mclean, J. m. (ig68) Trans. Amer. Acad. Ophthal.

Otolaryng., 72, I9I and McLEAN, J. M. (1966) Ibid., 70, 202

(1969) Amer. 7. Ophthal., 67, 477

$\longrightarrow,-$, and Nano, H. (1964) Trans. Amer. Acad. Ophthal. Otolaryng., 68, $4^{12}$

M PHerson, A. (1968) "New and Controversial Aspects of Retinal Detachment: International

Symposium". Hoeber Medical Division of Harper and Row, New York

SHEA, м. (1968) Canad. F. Ophthal., 3, Io9 